\title{
Estimating Cortical Surface Motion Using Stereopsis for Brain Deformation Models ${ }^{\star}$
}

\author{
Hai Sun ${ }^{1}$, Hany Farid ${ }^{2}$, Kyle Rick ${ }^{1}$, Alex Hartov ${ }^{1}$, David W. Roberts ${ }^{3}$, and \\ Keith D. Paulsen ${ }^{1,3}$ \\ 1 Thayer School of Engineering, Dartmouth College, Hanover, NH 03755, USA \\ 2 Department of Computer Science, Dartmouth College, Hanover, NH 03755, USA \\ $\{$ Hai.Sun, Hany.Farid, Kyle.Rick, Alex.Hartov, \\ Keith.Paulsen\}@Dartmouth. edu \\ 3 Dartmouth Hitchcock Medical Center, Lebanon, NH 03766, USA \\ David.W.Roberts@Hitchcock.org
}

\begin{abstract}
We present a method for estimating the 3-D structure of the cortical surface using an operating microscope whose binocular optics are fitted with two CCD cameras. The cortical surface is then tracked over time and the measured displacement can be used to drive FEM-based models for the recovery of subsurface brain deformations. We show the efficacy of this technique on a clinical case.
\end{abstract}

\section{Introduction}

The conventional neuro-navigational system based on rigid registration is subject to inaccuracies caused by brain deformations [12]. Factors that cause such deformations include gravity, drug administration, loss of cerebral-spinal fluid (CSF), and tissue retraction and removal. In order to compensate for these deformations, several groups, including ours, have developed non-rigid registration techniques based on finite element methods (FEM) for updating pre-operative data 3[45]. In this paper we describe a methodology to capture, in near realtime, the motion of the cortical surface in order to improve the accuracy of these FEM-based models. The technique involves six basic steps:

1. from pre-operative MRI scans, generate a FEM mesh of the entire brain,

2. register this mesh with the real-time surgical scene (in our case, as viewed through an operating microscope),

3. identify corresponding matches between the mesh and the cortical surface (at this point we have rigidly registered the pre- and intra-operative data),

4. estimate the shape of the 3-D cortical surface,

5. estimate the displacement of the 3 -D cortical surface over time,

6. and incorporate the estimated motion of the cortical surface into the original FEM mesh to produce a full volume description of brain deformations.

* The project is funded by the National Institute of Neurological Disorders and Stroke (NINDS, R01-NS33900). Farid was funded by an Alfred P. Sloan Fellowship, a NSF CAREER Award (IIS-99-83806), and a department NSF infrastructure grant (EIA98-02068). 
In the related work of [6], Audette et. al. used a commercial laser range sensor (in phantom studies) to estimate the 3-D cortical structure, as opposed to our proposed passive imaging system. Skrinjar et. al. also utilized stereopsis, but with a weak camera calibration and a restrictive Lambertian assumption [7].

Portions of our proposed system (steps 1-2) have appeared in 89, but for completeness, we will describe the full system, and show results from a case study of a left temporal craniotomy, in anticipation of generating model updates of pre-operative MR.

\section{Methods}

\subsection{Finite Element Mesh}

The compensation for brain deformation begins with the generation of a full volume finite element mesh. A pre-operative MRI scan is first obtained and imported into the software ANALYZE 1. The cranium is removed through semiautomatic segmentation. The marching cubes algorithm (with appropriate levels of surface decimation) renders the remaining brain tissue parenchyma into a surface wire frame composed of surface nodes, which is then used to produce the complete mesh (see [8] for more details).

\section{$2.2 \quad$ Rigid Registration}

The coordinate space of the constructed finite element mesh (i.e., the preoperative space) is then registered to the 3 -D operating room and the 2-D operating microscope 2 (i.e., intra-operative space). This process requires a rigid-body registration between the pre-operative and OR spaces, calibration of the microscope optics, and tracking the patient and microscope throughout the surgery (see [109] for more details).

\subsection{Mesh to Cortical Surface Registration}

With the pre-operative mesh registered to the microscope image, we now need to identify the portion of the mesh that corresponds to the exposed cortical surface after the craniotomy. The coordinates of the surface nodes are first projected into the microscope image. To avoid the inherent ambiguity resulting from this $3-\mathrm{D}$ to $2-\mathrm{D}$ projection, the surface nodes from only the hemisphere closest to the craniotomy are projected. The boundary of the craniotomy site is manually outlined in the microscope image. The surface nodes that fall within this boundary are identified in the microscope image and hence in the original mesh - we will refer to this subset of nodes as the craniotomy nodes. These steps constitute the rigid registration between the pre-operative mesh and the intra-operative microscope image.

\footnotetext{
${ }^{1}$ The software ANALYZE was provided by the Mayo Foundation.

2 The operating microscope, Model M695, Leica USA, Rockleigh, NJ.
} 


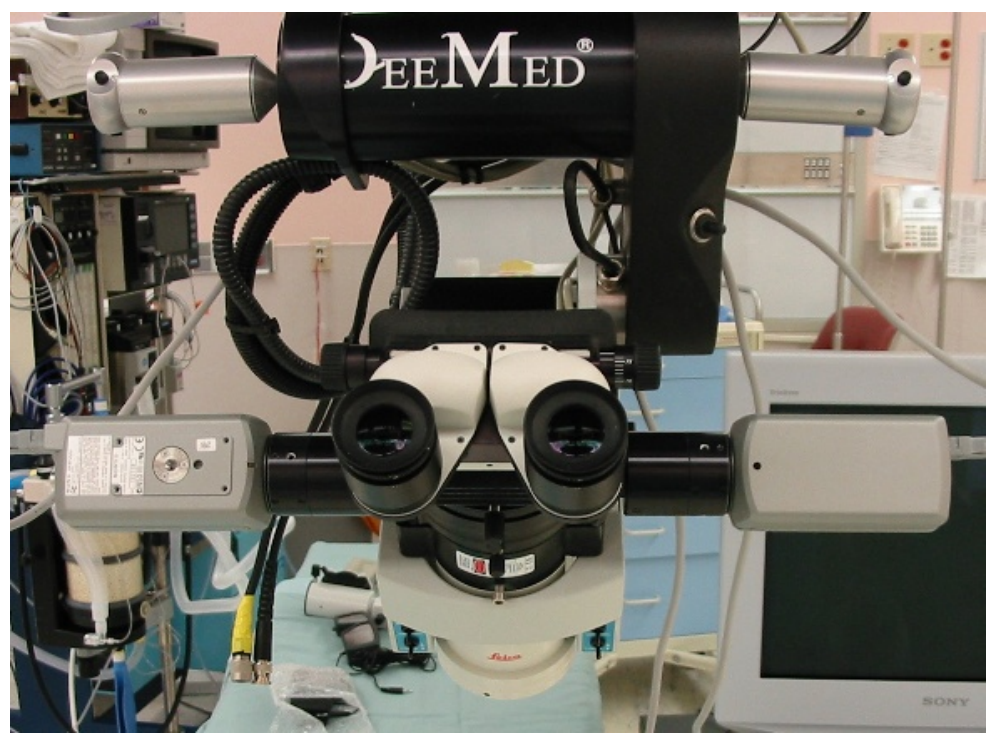

Fig. 1. Two CCD cameras have been attached to the binocular optics of the operating microscope. The resulting stereo image pair is used to estimate the shape of the cortical surface.

We next employ FEM-based non-rigid registration to compensate for brain deformations. In order to deploy these models effectively, we need to estimate the shape of the cortical surface, track the motion of the surface over time, and finally use this motion to guide FEM-based brain models.

\subsection{3-D Cortical Surface Estimation}

Stereopsis is employed to estimate the 3-D cortical surface. To facilitate surface estimation, two CCD cameras 3 have been attached to the binocular optics of the operating microscope, Fig. 1 A stereo image pair is acquired at 15 frames per second. The stereo images can be displayed on a computer monitor and fused using LCD glasses to provide a real-time 3-D view of the operating scene.

Estimating the 3-D surface involves two main steps: (1) for each point in the left stereo image, find the corresponding point in the right image (i.e., stereo matching). There is a significant literature on stereo matching (see, e.g., [11] for a general survey). Building on this literature we have implemented a number of matching constraints tailored to the recovery of a cortical surface (see [12 for more details); (2) from this correspondence, use triangulation to determine the 3 -D coordinates.

For computational considerations, we find correspondence between only a subset of the pixels. Specifically, the boundary of the cortical surface is manually

\footnotetext{
${ }^{3}$ Firewire CCD camera, Sony DFW-x700, Resolution $1024(H) \times 768(V)$, Sony Corp.
} 
outlined, and approximately 1000 uniformly spaced pixels within this region are reconstructed. A surface is then fit to these estimated 3-D coordinates to reduce sensitivity to noise and any incorrect correspondences (we use a spherical model to simplify the tracking as described next).

\subsection{Tracking the Cortical Surface}

Prior to opening the dura, the cortical surface location is estimated from the stereo pair as described above. At this point, we assume that brain deformation is minimal. After opening the dura, the cortical surface is re-estimated approximately every minute.

Employing rigid registration between the pre-operative mesh and the OR space (Section 2.2), we first localize the craniotomy nodes on the initial estimate of the cortical surface location. The subsequent tracking of the surface and craniotomy nodes is performed by using the iterative closest point algorithm (ICP) [13], and for comparison by using a "gravitational sagging" (GS) technique. In either case, the tracking involves estimating the cortical surface at time $t$ and $t+\Delta t$, fitting a spherical model to each surface, and then finding the motion of the craniotomy nodes (using ICP or GS) between these two surfaces. Tracking using ICP is performed by finding a correspondence between nodes that minimizes the overall Euclidean distances between their positions. Tracking using GS is performed by intersecting each node of the surface at time $t$ with the surface at time $t+\Delta t$ along the direction of gravity.

\subsection{Recovering Full Volume Brain Deformations}

Having obtained the motion of the surface nodes at the craniotomy site, we can use these data to guide the FEM-based model to recover the deformation for the entire brain. The integration of this data into a finite element model is a nontrivial problem and is currently under investigation by several groups including our own 31415.

\section{Results}

In this section, a case study is presented to illustrate the estimation of cortical surface motion for use in brain deformation modeling. The surgical procedure was a craniotomy for the removal of a cystic lesion and hamartoma in the left temporal lobe. Prior to surgery, an MRI scan was obtained, and the cranium segmented. A FEM mesh was generated from the resulting segmented brain (Fig.2(a)), and registered to the real-time microscope image, Fig. 2(b), acquired at the start of surgery. The surface nodes that correspond to regions of the craniotomy site are identified, Fig. 2(c). Fig. 2(d) shows the same FEM mesh in panel (a) but with the craniotomy nodes colored in gray.

Shown in Fig. 3 is the reconstructed cortical surfaces before and after cyst drainage. Shown in Fig $3(a-b)$ are microscope images before and after cyst 


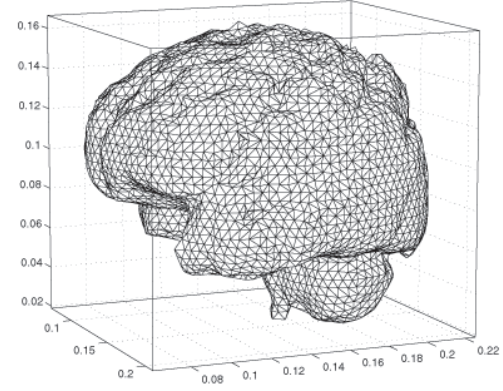

(a)

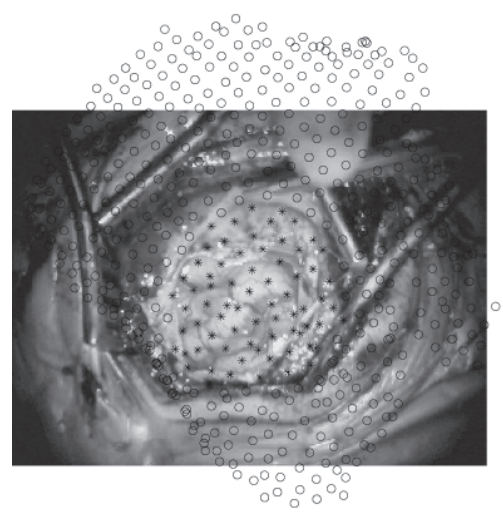

(c)

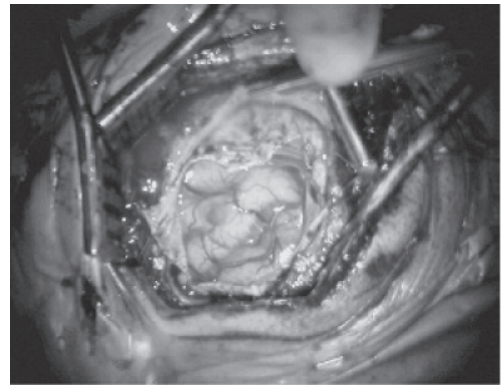

(b)

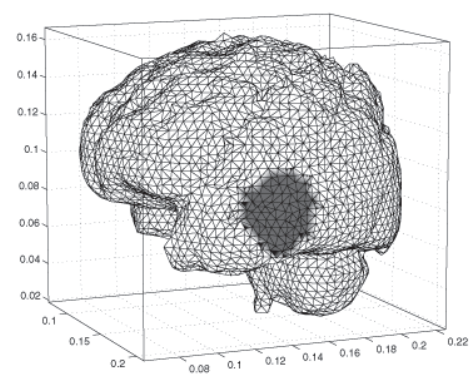

(d)

Fig. 2. Shown are: (a) the finite element mesh generated from the pre-operative data; (b) an image of the surgical scene viewed through the operating microscope; (c) the result of projecting the mesh surface nodes onto the surgical scene, where the nodes within the craniotomy boundary are plotted as asterisks and the others as open circles; and (d) the mesh with the craniotomy nodes colored in gray.

drainage, respectively, while Fig 3(c-d) contains the estimated cortical surface from two different vantage points. Note the significant flattening and shift of the cortical surface post-drainage. The micro-screw fiducials (BMs) are marked in the microscope images and plotted in the reconstructed cortical surfaces for orientation purposes. Running on a $1.1 \mathrm{GHz}$ machine, the estimation of the cortical surface for a $1024 \times 768$ stereo image pair requires approximately 60 seconds of computation.

To quantify the accuracy of the estimated cortical surface location, we measured, using an optical stylus probe, the 3-D location of several micro-screw fiducials placed in the bony rim of the cranial opening ${ }^{4}$. These fiducials are denoted as BMs in Fig. 3. These 3-D locations were compared to the corre-

${ }^{4}$ Leibinger E-Z Flap Plating System, Styker Cooperation, Kalamazoo, MI. 


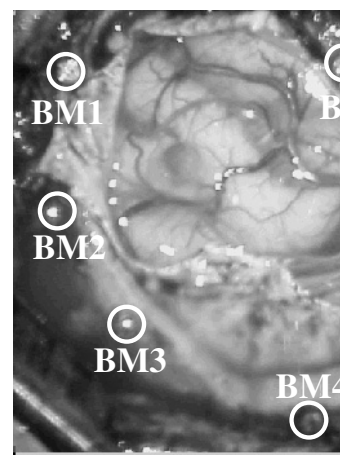

(a)

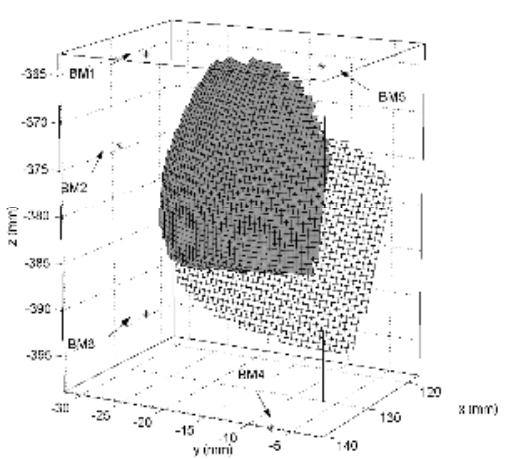

(c)

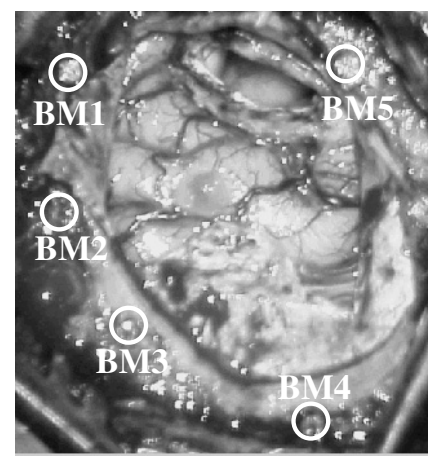

(b)

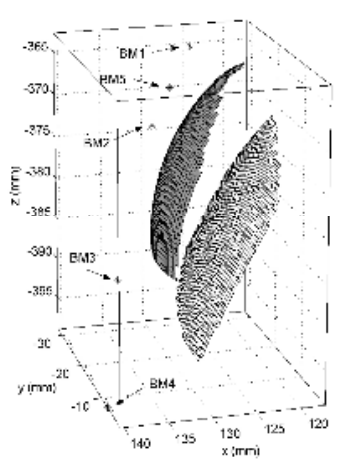

(d)

Fig. 3. The reconstructed cortical surface before and after cyst drainage. Shown are: (a) the surgical scene before cyst drainage; (b) the surgical scene after drainage; and $(\mathrm{c}, \mathrm{d})$ the reconstructed cortical surfaces before (gray) and after (white) drainage. The micro-screw fiducials (BMs) were marked in the microscope images and were plotted with the reconstructed surfaces for orientation purposes.

sponding 3-D locations using stereopsis - the center of each micro-screw was manually identified in the microscope images and its 3-D location was computed as the mean of the 3 -D coordinates of a small neighborhood centered about this position. The absolute difference between these estimates is $1.6 \mathrm{~mm}$ with a standard deviation of $0.9 \mathrm{~mm}$. The maximum error is $2.7 \mathrm{~mm}$, with a minimum error of $0.9 \mathrm{~mm}$.

To quantify the accuracy in tracking of the cortical surface, we again compare results obtained through stereopsis with optical stylus measurements. Shown in Fig. 4 are three cortical features (CF1, CF2, and CF3). Their locations were digitized using the stylus before and after cyst drainage. These locations were compared to the locations of the same features on the estimated cortical surface. When using stereopsis for tracking these cortical points, we manually locate them on the reconstructed surface before cyst drainage. The displacement of 

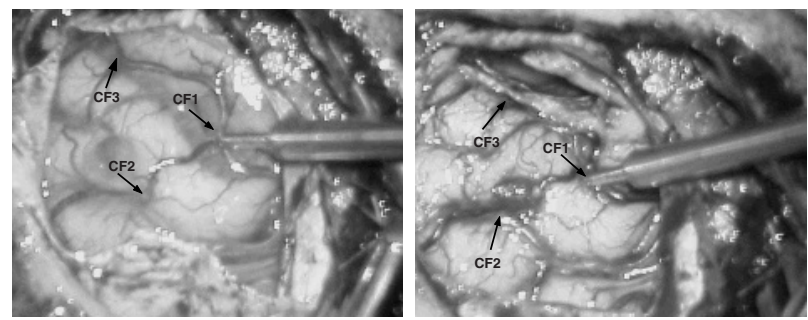

\begin{tabular}{r|c|c|c} 
& CF1 & CF2 & CF3 \\
\hline stylus & 11.6 & 8.9 & 11.8 \\
ICP & 9.3 & 7.5 & 9.7 \\
GS & 13.3 & 9.8 & 12.1
\end{tabular}

Fig. 4. The cortical features (CF1, CF2, and CF3) were digitized using the optical stylus before (left) and after (right) cyst drainage. Shown in the table are the estimated displacements $(\mathrm{mm})$ of these features from the image-based tracking methods (ICP and GS) relative to the stylus method.

each feature was then calculated using ICP as described in Section 2.5] and using a simple gravitational model (GS), also described in Section 2.5. Shown in Fig. 4 . are the estimated displacements for all three techniques. Note that, in this case at least, the ICP approach under-estimates and the GS method over-estimates the displacement measurements acquired from the stylus probe. While the stylus probe defines the world (OR) coordinate system, which is regarded as "ground truth", it also contributes an error component although if well calibrated the probe error is expected to be small. This error, however, may be exacerbated due to the inaccuracies in locating precisely the same position on the cortical surface before and after cyst drainage.

\section{Discussion}

We have presented a technique for estimating cortical surface motion for FEMbased brain deformation models. This technique promises to improve neuronavigational systems that are currently based solely on rigid-registration. The operating microscope employed is readily available in a neurosurgical OR. The estimation of the 3 -D cortical surface via stereopsis is efficient (at 1 frame per minute) and avoids any contact-induced tissue deformation. The estimated cortical surface is, on average, accurate to within $1.6 \mathrm{~mm}$, and the tracking of the cortical surface to within $1.5 \mathrm{~mm}$.

We are currently investigating several extensions and improvements to this work. We would like to design and implement phantom experiments in which quantitative validation of our approach can be obtained. We also plan to explore other, computationally efficient yet accurate, parameterizations of the cortical surface that go beyond the simple spherical model. In order to improve the tracking, we plan to replace ICP and GS with more sophisticated tracking algorithms that allow us to correlate features in the microscope image with the reconstructed cortical surface. Finally, we are working to incorporate the motion of the cortical surface into the FEM model to update the pre-operative MR. 


\section{References}

1. Hill, D., Maurer, C., Maciunas, R., Barwise, J., Fitzpatrick, J., Wang, M.: Measurement of intraoperative brain surface deformation under a craniotomy. Neurosurgery 43 (1998) 514-528

2. Roberts, D., Hartov, A., Kennedy, F., Miga, M., Paulsen, K.: Intraoperative brain shift and deformation: A quantitative analysis of cortical displacement in 28 cases. Neurosurgery 43 (1998) 749-760

3. Ferrant, M., Nabavi, A., Macq, B., Jolesz, F., Kikinis, R., Warfield, S.: Registration of 3-d intraoperative mr images of the brain using a finite-element biomechanical model. IEEE Transactions on Medical Imaging 20 (2001) 1384-1397

4. Paulsen, K., Miga, M., Kennedy, F., Hoopes, P., Hartov, A., Roberts, D.: A computational model for tracking subsurface tissue deformation during stereotactic neurosurgery. IEEE Transactions on Biomedical Engineering 46 (1999) 213-225

5. Škrinjar, O.: Deformable Models in Image-Guided Neurosurgery. PhD thesis, Yale University, New Haven, CT (2002)

6. Audette, M., Siddiqi, K., Peters, T.: Level-set surface segmentation and fast cortical range image tracking for computing intrasurgical deformations. Medical Image Computing and Computer-Assisted Intervention - MICCAI'99 (1999) 788-797

7. Skrinjar, O., Tagare, H., Duncan, J.: Surface growing from stereo images. IEEE Computer Society Conference on Computer Vision and Pattern Recognition (CVPR 2000) (2000) 571-576

8. Sullivan, J., Charron, G., Paulsen, K.: A three-dimensional mesh generator for arbitrary multiple material domains. Finite Elements in Analysis and Design 25 (1997) 219-241

9. Sun, H., Farid, H., Hartov, A., Lunn, K., Roberts, D., Paulsen, K.: Real-time correction scheme for calibration and implementation of microscope-based imageguided neurosurgery. Proceedings of SPIE Medical Imaging 2002, Visualization, Display, and Image-Guided Procedures 4681 (2002) 47-54

10. Edwards, P., King, A., Jr., C.M., Hawkes, D., Hill, D., Gaston, R., Fenlon, M., Jusczyzck, A., Strong, A., Chandler, C., Gleeson, M.: Design and evaluation of a system for microscope-assisted guided interventions (MAGI). IEEE Trans. on Med. Imaging 19 (2000) 1082-1093

11. Dhond, U., Aggarwal, J.: Structure from stereo - a review. IEEE Trans. on Systems, Man and Cybernetics 19 (1989) 1489-1510

12. Sun, H., Roberts, D., Hartov, A., Rick, K., Paulsen, K.: Using cortical vessels for patient registration during image-guided neurosurgery. Proceedings of SPIE Medical Imaging 2003, Visualization, Display, and Image-Guided Procedures (IN PRESS) (2003)

13. Besl, P., McKay, N.: A method for registration of 3-D shapes. IEEE Trans. on Pattern Analysis and Machine Intelligence 14 (1992) 239-256

14. Skrinjar, O., Nabavi, A., Duncan, J.: Model-driven brain shift compensation. Medical Image Analysis 6 (2002) 361-373

15. Lunn, K., Paulsen, K., Roberts, D., Kennedy, F., Hartov, A.: Inverse technique for combined model and sparse data estimates of brain motion. Proceedings of SPIE Medical Imaging 2003, Visualization, Display, and Image-Guided Procedures (IN PRESS) (2003) 